Article

\title{
Undecidability and the Evolution of Ideas in an Emergency Event: An Example of How to Systemically Test Organizational Effectiveness (OE) in University Groups
}

\author{
Romina Fucà $1, *(\mathbb{D})$ and Serena Cubico ${ }^{2, *(\mathbb{D})}$ \\ 1 Department of Law and Communication Science, University of Macerata, 62100 Macerata, Italy \\ 2 Department of Business Administration, University of Verona, 37129 Verona, Italy \\ * Correspondence: romina.fuca@hotmail.it (R.F.); serena.cubico@univr.it (S.C.)
}

Received: 25 March 2020; Accepted: 7 May 2020; Published: 11 May 2020

\begin{abstract}
The location of this research is the university, through which we are progressively channeled into a seemingly insoluble Gordian knot. What is our participation in the university and what cultural and human commitments inform this participation? More trivially, what rights and duties does the individual acquire or lose within his or her academic identities? Our main target was finding an ideal organizational practice to examine, such as an emergency event. What strategy can the university adopt? Can it realign its distortions and retain its resources? How and in what ways? What information is needed for this purpose? Which actors are relevant in this process? A systemic survey model is, therefore, presented to analyze data obtained from a sample of 200 respondents from various academic groups, including students, professors, administrative staff, and other stakeholders. Quotas were used for the primary challenge posed by the pictures representing dimensions according to a systemic schema of organizational effectiveness (OE). Respondents were then asked to judge the dimensions and pictures against their personal capacity for intellectual identity, functionalism, and materialism. During the test, the participants were expected to develop their capacity to approach phenomenal consciousness and the search for its neural correlates, thereby becoming familiar with the high-order demands and challenges posed by the current information available to them. A nine-item interval behaviorally anchored rating scale (BARS) was used to develop a systemic matrix that could show the participants' collective OE when an emergency event occurs at the university. This study aims to stimulate a broader investigation into the preparation of programs and plans that should be a priority today in the context of sustainability in educational institutions, thereby setting useful thresholds on decision-making paths. To develop the collective model, a matrix generated by each respondents' dimensional modal values (DMVs) in the test and the overall samples' modal values (OMMVs) were used. Borrowing from Luce's theory of probability, we analyzed the similarity of the OE university matrix from the results in descending order, restricting our attention to modal values which were chosen for the test and demonstrate how the learning model was formulated to assume that each group with evolved behavior could respond adaptively to a conditional function thanks to its permanence in a university environment.
\end{abstract}

Keywords: European universities; general systems theory; self-reference; emergency events; issues on collective decision-making; behaviorally anchored rating scales (BARS); theory of decision; discrete choice under uncertainty; organizational effectiveness 


\section{Introduction}

A "natural disaster" does not amount to a "violation of human rights", the latter being increasingly condemned by the intersection of the universalist and trans-contextual. The concept of "contextualism" has been promoted by D. Miller, among others [1]. An emergency event often requires a slightly more relaxed attitude to the equality of distance. We are not talking about discrimination, nor a sanction of well-being, but a more formal universalism that focuses on substantive issues.

Who has more rights in emergencies, and when does a distinction occur that makes a groundbreaking principle arbitrary? Reduction cannot only concern economic variables because it is also, at present, a complex organizational practice of university governance that is able to restrict and/or cut courses, faculty members, and even academics. Governance has the challenging task of bringing the structure and the staff of the university into conformity, as well as managing the participation and maintenance of initiatives co-funded by the European Union or other third parties, such as foundations and companies, etc.

It seemed appropriate to analyze the steps for the generation of a decision tree which can sketch the structure of organizational effectiveness (OE) through which the four university groups of respondents can operate with an integrated approach during an emergency. At first glance, this decision tree is considered "bushy" because it entails a high degree of complexity [2].

We believe that in the case of an important subject, such as that of an emergency event, the general attitudes aroused by personal relationships within academic groups are capable of expressing appropriate psychological aspects, such as opinions and information sharing. We also hope that these academic groups will be "sensitive" and not "apathetic" towards environmental issues, and that their actual behavior will be able to distinguish between those who are uninterested in the impacts of organizationally and institutionally redefining events and those who are optimistic, even when they are not overly apprehensive (in a way that might evenly be counter-effective).

We also theoretically asked ourselves the following questions: (1) Are the four groups of university respondents independent?; (2) Do some options have others as a prerequisite?; (3) Are there potential synergies or dyssynergia?, for instance, Are there options that would likely work better than others that were also selected?, or, alternatively, Are there options that are broadly similar and therefore offer replicates rather than real benefits?

We found that, among certain micro-constellations of social actors and institutional agents that make decisions with narrow resources among themselves, these questions not only highlight the possibility of diverging priorities and values but also the limits of mandates and the misalignment of information sharing. Negotiation, the formation of coalitions, increases in power, and the mobilization of actors who are not considered to be part of the system entail a type of academic "egalitarianism" beyond the possibility of considering such factors.

We question whether the institutional and statutory bodies of the university at large can also empower people to design, implement, and extend the decision-making process from the perspective of their predisposition to share an ideal university system.

Our observations are indeed conditional on the processes and parameters of choice in an "interdependent spatial environment" (that of the university), including variables with latent and random effects. We integrated suggestions based on a qualitative understanding on this collective issue through the creation of four practical choice samples to model the effectiveness of the four response sets at the university.

\subsection{Prior Considerations to Design Decision-Making in the Event of an Emergency}

Our first stage model was constructed to visualize the distributions or utility functions in our model that could articulate decision-making according to classical decision theory, as well as facilitate an innovative inquiry onto the psychological aspects of how humans are able to cope with emergencies related to substantial questions. We examined, for instance, a general emergency plan and found that various models and variants were fundamental to observational systems, such as university 
academies and colleges, which presented the most recognizable face of the Zeitgeist (the spirit of the time) but at the same time shared too much information to be observed in a balanced manner in their forms of pluralistic integration and local terminals through partial dialogue, which was sometimes immeasurable.

The right to assist academic institutions during emerging events, while preserving past institutional orientations, would also require the transfer of resources from certain strategic actors to others, since the evaluation of any preexisting vulnerability, as well as the exposure threshold, varies from one individual to another.

Organizational preparation activities include:

- Developing emergency response plans;

- The training of employees and ad hoc staff on what to do in an emergency;

- The acquisition of the necessary equipment, tools, and materials;

- The implementation of training and exercise tools.

In terms of response and recovery, institutional capacity plays a central role. For instance, the effectiveness of a disaster risk management system depends on the knowledge, awareness, and capacities of the stakeholders involved, including central governments, local actors, and smaller communities.

The "contingent approach" highlights the possible means of differentiation between alternative forms of organizational structures and management systems. There is no optimal state. In essence, the structure of an organization and its success are dependent - that is, contingent-on the nature of the tasks that are designed to be handled, as well as the nature of the environmental influences. This theory is based on the assumption that group results are a function of the combination of: (a) the questions asked about the group and the resources that group provides; and (b) the communication processes that the group employs to answer these questions and use its resources.

Our general guideline is, instead, the theory of the general system (GST), which, from its first authors to its most recent examples, offers a meta-methodology that seeks to embrace different sciences and methodologies by always engaging with several concepts, laws, and models that are applicable (with due diligence) to systems of all types.

We assume the presence of an emergency event where: (1) recovery is comparatively the most effective solution; and (2) recovery is most effective when more organized and efficient institutions are involved. Our theory also assumes that: (3) the respondents of the samples act in the public interest of the university; and (4) information about the relevant problems and consequences is widely disseminated. Finally, it is assumed that: (5) the respondents know the general university structure. The core of these basic assumptions is captured in Figure 1.

We found that, in some of these micro-constellations of social actors and institutional agents, making a decision using resources that are themselves poor involves highlights not only the possibility of diverging priorities and values, but also the limitations of such mandates and misalignments in the sharing of information. Negotiation, coalition formation, increases in power, and the mobilization of actors who are not considered part of the system, then, assumes a kind of university "egalitarianism" which extends beyond the technical possibility of facing an emergency event. We, therefore, asked ourselves whether the institutional and statutory bodies at a university can also empower people to design, implement, and disseminate decision-making processes, and whether these abilities can be increased from the perspective of their ability to enhance community rescue. 


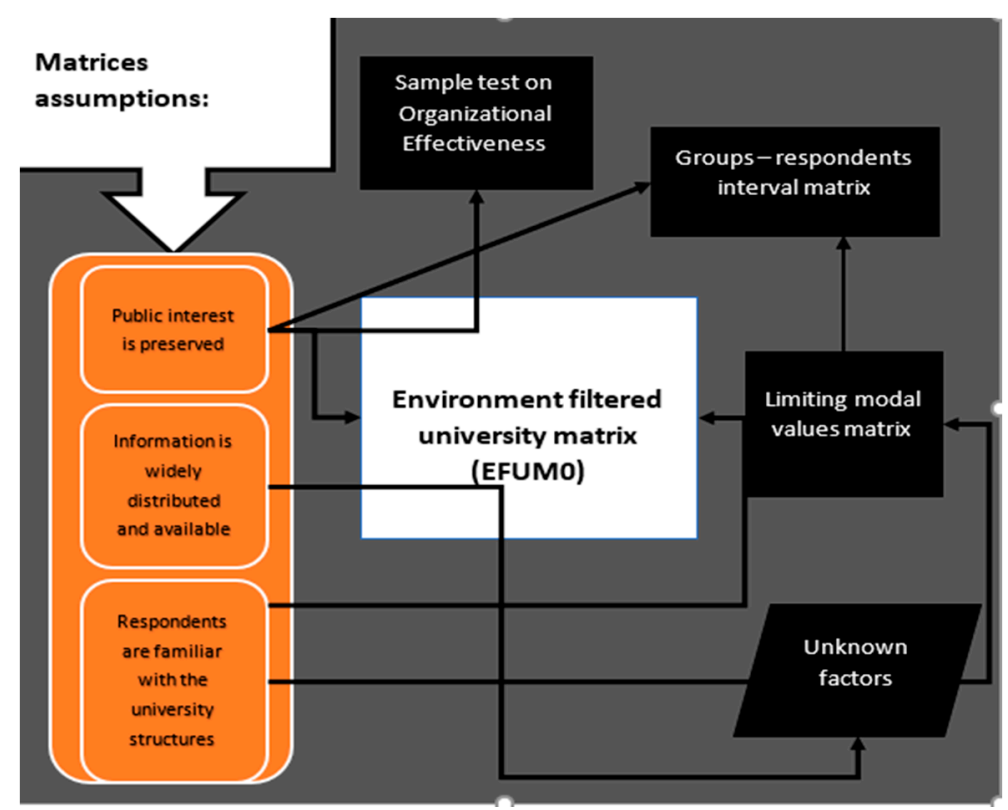

Figure 1. Matrix assumptions used to create an organizational effectiveness (OE) model in the event of an emergency at a university.

\subsection{Recovery and Institutional Capability}

An important assumption for the purpose of our discussion is that the efficiency and effectiveness of both routine and emergency response activities are generally facilitated by the preparatory actions undertaken at the community, organizational, and national levels. Since companies with complex organizational models require particularly complex organizational models for non-routine events, such as an emergency, an assessment of preparedness and response activities requires a prior understanding of the complex issues these units may face, the tasks they must perform, and the ways in which they will mobilize their resources [3,4].

Notably, the rigid link between the evaluation and outcome measures at the university level has often been criticized because this assumption may penalize the actors for their bad luck and reward them for good luck while ignoring their true contributions [5] (p. 543). This can happen, for example, because the effectiveness of an organization like the university must make use of bodies at the institutional level, such as government commissions and university senates and councils, and these, in turn, can also be composed of internal or external actors (or stakeholders) and include representatives of the two groups. In so doing, they can use both outcome measures and their own situational awareness.

As expressed among others by K. A. Merchant et al. (2007), subjective assessments are likely to be subject to distortion. One bias is known as the outcome effect. An experimental study in this area found that making results known to evaluators significantly influenced performance assessments even when the outcome measures were not aimed at the significance of individuals' performance. Another relative blame is known as the hindsight effect. Research on the hindsight effect shows that evaluators with knowledge of the results tend to take information about the pre-result circumstances that were not available for those who were evaluated [5]. These hypotheses prejudice the pre-result probabilities and, hence, their evaluations.

In addition, as A. Sajo (1981) notes, since the administrative organization operates in a defined social environment, and its agents are not isolated and also live in their own formal organizations, "it often happens that the organization or its individual members act in accordance with environmental influences and use or even break regulatory loopholes" [6] (p. 74). In some cases, this can be beneficial for the organization because it makes adaptation and innovation possible and is therefore coupled with 
the tacit approval of the "seniors", especially if this does not entail a violation of the formal prevalence of the law.

\subsection{Is There Someone at the University Who Has More Rights Than Others in the Event of an Emergency?}

In the case of an emergency event at a university, another question to ask is how the roles of the four groups (students, professors, administrative staff, and interveners) may be extended when assessing their response to decision-making. Do these roles include a specification of how the options should be assessed (that is, what factors should be considered) or do they simply assess their options in light of an established program?

When a routine response is not possible, management measures should be improvised, even if the organizational level (influenced not only by internal structures, systems, and procedures, but also by the collective capacities of staff and through the broader offshore institutional environment) can offer a spectrum of ways in which complex and interdependent issues can be treated as elements of collective interest.

The history of experience with multi-level governance institutions affects the ways that local participants understand, implement, modify, or ignore the rules written by external officials $[7,8]$. For example, when all participants share a set of common values and interact with each other in a set of multiplex arrangements within a small community, their chances of developing adequate rules and standards to govern repetitive relationships are much greater [3,7].

As Smithson [3,9] points out, education often operates through disinformation (for example by promoting a false impression of approval) or by referential abbreviation (in particular, imprecision and ambiguity, as in expressions of alleged delicacy). In their volume on communication errors, Mortensen and Ayers (1997) clearly align themselves with "the ideal of maximum communicative value-clarity, fluidity and clarity", but they are obliged to recognize that there are many occasions when abuse and even deception are socially acceptable [10].

People may have somewhat reasonable grounds for ignoring information that directly affects them, even when that information is readily available [11]. The rate of genetic testing for the risk of inherited life-threatening disease like Huntington's chorea or colon cancer is notoriously low, and the same goes for diagnosing the evolution of these conditions [12]. Less dramatic examples include the majority of parents who do not want to know the genders of their unborn children, social aspects like being surprised by a gift, entertainment (for example, to avoid prematurely learning the end of a novel or a film), and games [13].

One principle of decision-making is that the actors build their problems not from "real problems" but from a mixture of the systematically recognized characteristics of "the space of problems". However, in this procedure, such problems might not be "real enough" for the actors but only "realistic", with the consequence that "already in the analysis of the conditions of the possibility of observing [the actors may] enter a problem that is repeated in the analysis of the decision. How is it possible to use a distinction, but to indicate only one side, that is to say without indicating the distinction itself?" [14] (p. 104).

Another element of contingency is added by the frequent recourse to "experts" for whom the questions are: What types of political relations do they have?; Are they sure they don't fall into performative self-contradictions?; and Do they agree with the leading direction or could they, on the contrary, trigger changes to respond within a more efficient system?

According to J. P. Olsen (2005), the university "as an institution is bound by a pact with long-term cultural achievements". In fact, the university is a trust system and those who belong to it are supposed to be the guardians of its purposes, principles, and constitutive processes. These actors are supposed to defend institutional identity and integrity, whether the threats come from outside or from within. Third parties are also supposed to strengthen rules and sanction non-compliance with institutionalized codes [15] (p. 5). 
Authority is undoubtedly a necessity for the positive achievement of organizational goals, but it will have to be responsible for both constitutional review and exposure to irregular and fluctuating waves of disobedience. In some cases, probability judgments are also formed based on a priori considerations.

In this context, Knorr Cetina (1999) introduced the term "negative knowledge", which describes our knowledge of the limits of knowledge, the amount of mistakes made in our ability to know, the things that we infer without knowing, and what people do not want to know. If we are to understand how people move towards uncertainty, then we must take these distinctions into account: "How can we assess the distinctions that are important to be made?".

However, this process seems to be limited to very special contexts such as that of a gambling house. In practical matters and in everyday life, there is generally no possibility of such a priori judgments. Finally, the recognition of vagueness in preferences and the realistic limitations of human reason directly yield the "partially ordered preferences in which the relation of indifference is no longer transitive" [16] (p. 4).

How E. Ostrom (2005) states that groups of individuals that work can exist in simple or complex nested systems ranging from very small to very large. The problem is that in a complex and interdependent world, effective organization is needed at all levels, from the smallest working group to international organizations. If the size of the group that governs and reaps the benefits is too small, negative externalities are likely to occur. Additionally, even in small face-to-face groups, some individuals can use a wide range of asymmetries to take advantage of others [8].

\section{Real World, Organizational Effectiveness, and Sample Space: Statistics at Work}

Decision theory, as the name suggests, concerns the problem of decision-making. The theory of statistical decisions consists of making decisions in the presence of statistical knowledge, which sheds light on some of the uncertainties linked to the decision problem. We assume, for the most part, that these uncertainties can be considered unknown numerical quantities and represent them with $\theta$ (possibly a vector or a matrix).

Conventional statistics aim to use "sampled information" (data obtained from statistical surveys) to make inferences about $\theta$. These conventional deductions are, for the most part, made regardless of their intended use. Decision theory, on the other hand, seeks to combine sampled information with other relevant aspects of the problem in order to make the best decision. In addition to the information sampled, two other types of information are generally relevant. The first is knowledge of the possible consequences of decisions. Often, this decision can be quantified by determining the loss that would occur for each possible decision and for the different possible values of $\theta$.

Ironically, knowledge often hampers decision-making. We understand that making a decision will entail risks and delay this action pending the advice of others or the possibility of processing other relevant information before choosing alternatives and assessing the consequences. Cooperating with other people especially highlights the expectation that additional support will improve our general judgments.

The discrete probability distributions indicate that if there is probability $p$ that an experiment will lead to result $A$, then if we repeat this experiment a large number of times, the fraction of times that $A$ will occur will be $p$. The set of possible results is called "sample space" [17].

As another example, a continuous probability distribution (PDF; or the density of a continuous random variable) is applicable to scenarios in which the set of possible outcomes can take values over a continuous interval-for example, real numbers, such as the temperature on a given day-which is generally described with the probability that each result is equal to 0 . For example, the probability that a given object weighs exactly $500 \mathrm{~g}$ is zero since the probability of measuring exactly $500 \mathrm{~g}$ trends to zero, which increases the precision of our measuring instruments [18].

Many problems in probability theory require us to count the number of ways in which a particular event can occur. For this, we study the themes of permutations and combinations. An activity must be carried out in a sequence of $\mathrm{r}$ phases. There are $n 1$ ways to perform the first phase; for each of these $n 1$ 
channels, there are $n 2$ channels for performing the second phase; for each of these $n 2$ ways, there are $n 3$ ways to perform the third phase, and so on. Thus, the total number of ways in which the whole activity can be carried out is given by the product $N=n 1 \cdot n 2 \cdot \ldots \cdot$ No [17].

The probability of the occurrence of two dependent events is the product of the "probability of the occurrence of one of them" for the "probability of the other occurring", when the first is considered to have taken place; the same rule extends to the occurrence of as many events as can be attributed. This inference may concern certain components of $\theta$, such as "(What is the value of $\theta 1$ ?)" or "(Is $\theta 2$ greater than $\theta 3$ ?)".

A distinction is often made between estimation and testing problems, depending on whether those problems affect the exact value of the parameters (or certain functions of the parameters) or simply offer a hypothesis concerning these parameters $[19,20]$.

More generally, inference covers the random phenomenon directed by $\theta$ and, therefore, includes prediction. For example, the evaluation of the distribution of a future observation $y$ depends on $\theta$ (and probably on the current observation $x), y \sim g(y \mid \theta, X)$. These divisions are sometimes artificial because all inferential problems can be expressed as estimation problems when considered from a theoretical point of view $[19,20]$.

In addition, the "invariance principle" states that if two problems have identical formal structures (i.e., if they have the same sampling space, parameter space, density, and loss functions) then the same decision rule should be used for both problems. This principle is used for a given problem by considering the transformations of the problem (for example, the changes of scale in the units of measurement) that involve transformation problems of an identical structure.

Gouran et al. (1993) argue that the probability of an appropriate choice in a decision-making or problem-solving discussion depends on whether the interaction allows members to:

- Show a good understanding of the problems to be solved;

- Determine the minimum characteristics that each alternative must have to be acceptable;

- Identify a set of relevant and realistic alternatives;

- Carefully consider the alternatives to each previously agreed characteristic of an acceptable choice;

- Select the alternative that the analysis suggests is most likely to have the desired characteristics [21].

Seven hypotheses underlie this set of claims. Since the presumed conditions are not applicable in specific examples, the probability that a group will be able to meet the requirements suggested by the declarations is greatly limited. The assumptions are as follows:

Members of a decision-making or problem-solving group are motivated to make an appropriate choice;

- The choice available is not obvious;

- The group's collective resources in relation to the particular task exceed those of the individual members;

- The requirements of the task are specifiable;

- Relevant information is available to members or can be obtained;

- The task falls within the intellectual capacities of the members who perform it;

- Communication is instrumental.

According to Scheidel and Crowell (1964), members of the group should engage in test-type models in which discussions revert to previous deliberations to change ideas and test new proposals [22] (p. 147). By agreeing on a question, the results accidentally emerge through role conflicts, factual analyses, and the anchoring of ideas in this cyclical movement.

Bayesian decision theory considers an ideal case in which the probability structure underlying the categories is perfectly known. This approach is based on a quantification of the trade-offs between the different classification decisions using the probability and the costs that accompany these decisions [23] (p. 31). 
There are generally two Bayes typologies that meet our needs:

- The Bayesian inference for binomial proportion;

- The Bayesian Gamma/Poisson model.

According to the Bayesian inference for the binomial proportion, the results of this phenomenon (or the ways in which the results are grouped) can take only one of two values: $A$ or $B$. Phenomenon $Z$ (any phenomenon with a "this or this" outcome) can be mathematically modeled as a random variable $Y$ with a binomial probability distribution of $p(Y)=(N Y) \theta Y(1-\theta)(N-Y)$, where $N$ is the number of tests (the total number of cancer patients, the shots, and the scores expressed), $Y$ is the number of successes or cases in which the result is equal to $A$, and $\theta$ is the unknown value between 0 and 1 that is equal to the proportion $Y / N$. This distribution describes the probability that $Z$ is equal to $Y$ in $N$ events (also called tests) of $Z$ [24] (pp. 107-112).

For the Bayesian Gamma/Poisson model, the posterior expectation can be considered as a dual solution to the mean square error (minimum mean square error, MMSE). In classical inference, there is no uniform solution (whatever the parameters) that is optimal for MMSE estimation. What exists in some cases is an unbiased estimator of uniformly minimal variance (UMVUES). The condition of impartiality guarantees solutions in cases where sufficient statistical parameters are involved and where the distribution function belongs to the exponential family. When UMVUES exists, subsequent Bayesian expectations are likely coincident or slightly distorted [24].

As expressed by E. Paté-Cornell (2007), probabilistic methods "presuppose the separation of probabilistic evaluations on the one hand, and preferences between the results of the scenarios on the other hand" [25] (p. 225). To be accurate in the risk analysis, if any decisions involve different similar systems and different time periods, the full distribution of that probability (per unit of time) cannot be reduced to its expected value and used as if there was not uncertainty about it. This is true because if $p(F)$ is the probability of failure in a given period of time, and if there is no uncertainty about it, the probability of $n$ independent failures in the same period is simply $[p(F)] n$. But if there are uncertainties regarding $p(F)$ (e.g., due to uncertainties regarding different parameter values), one can represent this probability as a random variable as described above. Even if the Bayesian probability of an event is the expected value of that random variable $(E V[p(F)])$, the probability of many of these independent events is generally not $E V[p(F)] n$, that is, the value one would obtain simply by increasing the probability of a single event to $n$ power. In that case, one cannot simply calculate the probability of an event (or a known number of events) as a single number (an average) and increase it to the power of $n$, even if one knew that number at the time analysis [25] (pp. 232-233).

\subsection{Situational Effectiveness in the Real World vs. Organizational Effectiveness in the Test}

The reality of the decision-making process lies between the decision-making processes that take place within organizations at different levels and with different subjects and those that relate accidentally (or even intentionally) to other decision-making processes that are not explicitly expressed in opposition to another decision in progress or do not correspond to a well-defined period, such as the ones we have taken into account-see Figure 2. on the following page.

Our general guideline is the theory of the general system (GST), which offers a kind of meta-methodology that seeks to embrace different sciences and methodologies by always following several concepts, laws, and models that are applicable (with due diligence) to systems of all types.

Decision-making is not necessarily rational or intentional but is limited by time and space. Relevant communication should take place during meetings among all members of the group but not outside the group [26]. This model distances itself from, for example, "good faith orientation" because the emergence of a decision focuses on how decision-making constitutes group boundaries and develops connections with the environment. 


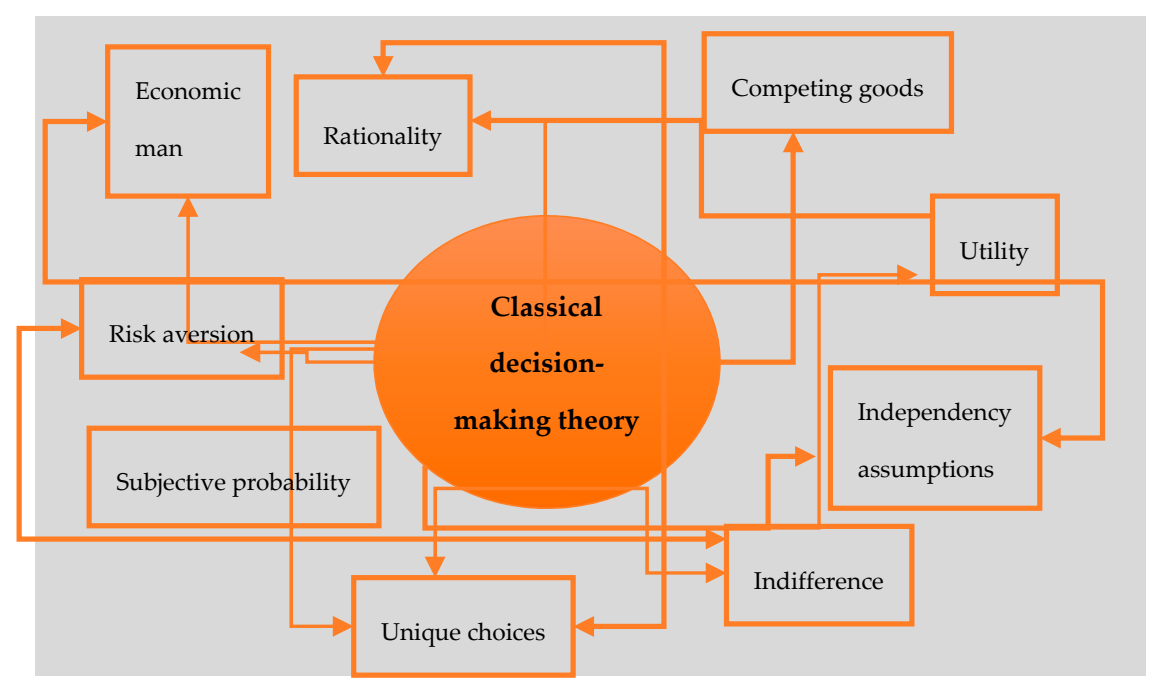

Figure 2. How people can decide according to the classical decision-making theory.

The focus shifts from "how group members deal with a problem" to "how decision-making processes relate to the group environment". For example, questions about the relevance of a problem can trigger renegotiation and a redefinition of group autonomy, which changes the cycles and stages of internal decision-making [22]. Likewise, changes in the group environment (for example, budget cuts, crises, and market changes) can change the ways members deliberate and use resources.

According to Price (1972), the traditional method for studying effectiveness has been the "objective approach" [27]. Objective, goal, mission, and task are all terms used to describe the capacity of an organization to exploit its environment in the acquisition of rare and precious resources in order to achieve its results.

Moreover, within academic institutions, as noted by the German sociologist N. Luhmann (2000), often "we do not choose", but "someone else chooses" because the target is not necessarily "a competition which boosts to higher levels the strategy of negotiation of the decisions instead of another" [28]. We must therefore ask ourselves, who are the real key players in the university process? What are their common interests and areas? Finally, are there common goals that emphasize cooperation? [29].

\subsection{The "Analysis of Inverse Preferences" and the BARS Graphics Test}

Thirty-three variables of $\mathrm{OE}$ issues previously identified by the researchers were each paired with a three-dimensional sub-illustration (not visible to the respondents during the test) in the test. The respondents were asked to rate these variables as $1=$ high, $2=$ medium, $3=$ weak, or $99=$ no answer (the number was in bold for those who received the telematic test, with a cross on the number chosen for those who received a printed version by email) without any prior knowledge of the event unless the three images on reactions to an unexpected event could be found through common means of communication (especially the internet).

At this point, the respondents had to assign a qualitative score $(q s)$ of the feelings generated by the submitted images ranging from 10 to 90 (in the empty field displayed to the left of the images) at tens intervals, thus representing the importance that the variable in the object had for them after self-evaluating the meaning of the three images with this variable (for an completely "no response" answer, they were still asked to assign a score).

Through self-constructed behaviorally anchored rating scale (BARS), we derived four different indices for the evaluation of the 33 sub-dimensional variables in the 99 global elements of the OE. Each of the four was connected to the four different groups chosen to be studied at the university in the event of an emergency. As mentioned above, and in accordance with the implicit scope of this research 
effort, focus was placed on the variables selected from the vast literature of general systems theory that summarize the significant contributions of each sub-dimensional component of $\mathrm{OE}$, such as:

- Management/coaching;

- Searching for results;

- Planning and organization;

- Personal awareness;

- Organizational commitment;

- Communication;

- Confidentiality;

- Interpersonal relationships;

- Public relations;

- Conflict resolution;

- Motivation/initiative;

- Teamwork;

- Inclusive attitude;

- Orientation to success.

Quotas were used, with the main challenge posed by the images representing the dimensions (according to the general systemic scheme) of organizational efficiency. Respondents were asked to rate the dimensions and images in terms of their personal ability to discern identity, functionalism, and materialism. During the test, respondents were expected to develop the ability to approach phenomenal awareness and the search for its neural correlates, thereby becoming familiar with the higher order demands and challenges posed by the prior information available to them.

The students of medium-sized universities in Europe numbered around 60,000 (first year students), professors numbered around 3000, administrative staff around 10,000, and external stakeholders around 9000 [30].

We suggested that in the test respondents should immediately make a decision that defined the possibilities that can also change their knowledge parameters compared to real-world situations (displayed through drawings and images) with fragments that were in their minds, thus building their personal definitions of the conceptual models (the 99 dimensions on which the interview takes place). Moreover, the formulation of relevant systems of targeted activity can express problematic situations and make such problems a reality.

Table 1 shows how the quotas were collected in the sample.

By using "inverse preference analysis" [26] (see Section 2.1), we aimed to show the organizational behavior of the four chosen academic groups when an unexpected event occurs through an integrated measurement of organizational effectiveness.

After the choice of the image and related description, the respondents scored them with an interval ranging from 10 to 90 . This minimized the risk that the respondents assign higher scores to achieve a better result and was, therefore, a realistic OE measure by respondents in the context of an unexpected event, leaving four levels before and four levels after "normal apprehension". The novelty of the experiment using the well-known behaviorally anchored rating scale is that the qualitative score criterion was developed backwards by the respondent, as, before assigning the images a value, the respondents could not pre-emptively know what feelings the images will arouse in their personal representativeness of the 33 sub-dimensional variables in the 99 global elements of the OE. 
Table 1. Quota sampling of the four groups of university respondents. ${ }^{*}$ Note: The sample size was 2000.

\begin{tabular}{ccccc}
\hline Quota Sampling & & & & \\
\hline Control Attribute & Women & $\begin{array}{c}\text { Population } \\
\text { Composition } \%\end{array}$ & $\begin{array}{c}\text { Targeted } \\
\text { Sample \% }\end{array}$ & $\begin{array}{c}\text { Composition } \\
\text { Count }\end{array}$ \\
\hline Sex & Men & 80 & 80 & 800 \\
& Other & 100 & 100 & 1.000 \\
Total & & 20 & 20 & 200 \\
Age Group & $19-35$ & 200 & 200 & 2.000 \\
& $36-45$ & 50 & 50 & 500 \\
& $46-66$ & 80 & 80 & 800 \\
Total & 67 and older & 40 & 40 & 400 \\
University Group & & 30 & 30 & 300 \\
& & 200 & 200 & 2.000 \\
& s/Students & 70 & 70 & 700 \\
& d/Professors & 40 & 40 & 400 \\
& a/Administrative Staff & 80 & 80 & 800 \\
Total & es/External & 10 & 10 & 100 \\
& Stakeholders & 200 & 200 & 2.000 \\
\hline
\end{tabular}

This scoring system, which ranged from 10 to 90 with dozens of intervals, did not indicate the respondent's ability (this was clarified when the test was delivered).

In reality, these ranges indicate the following:

- 10: uncontrollable hyper-apprehension about the occurrence of an unexpected event;

- 20: worrying hyper-fear;

- 30: severe hyper-apprehension;

- 40: hyper-moderate apprehension;

- 50: normal apprehension;

- 60: normal interest;

- 70: light interest;

- 80: moderate apathy;

- 90: severe apathy.

As shown in Figure 3, the scores are the dimensional modal values (DMVs) that the four university groups used to scale the pictures and definitions of the chosen test based on their qualitative scores $(q s)$. Each DMV group is also compared to the modal value for the overall score matrix in each group (the OMMVs).

This result agreed with the sampling concerns about inference, such as the quotas in Table 1 , which represented $10 \%$ of the total quota. Thus, visualization through modal values ensured that non-probability sampling elucidated how the observations tend to focus. The measurements were first calculated as a systemic test for the first responder from each of the four groups. Then, the entire quota sample for each of the four groups was generated via a RAND().exe formula. The results were also normally volatile, so they were first fixed as values and then calculated based on their frequency of occurrence for each dimension of the OE. 


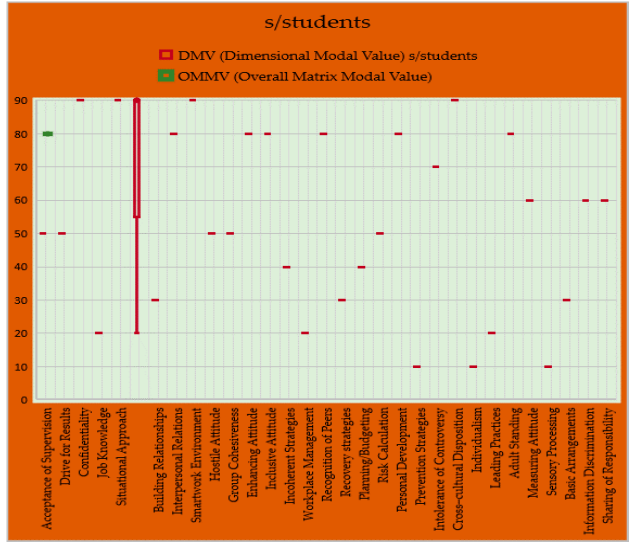

(a) DMVs for s/students

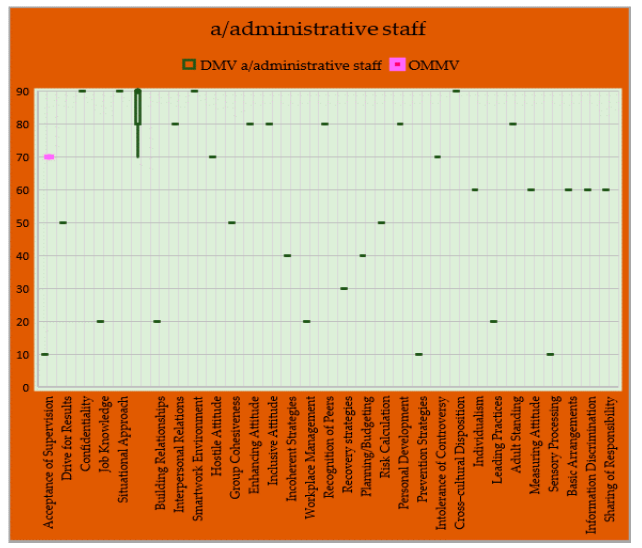

(c) DMVs for a/administrative staff

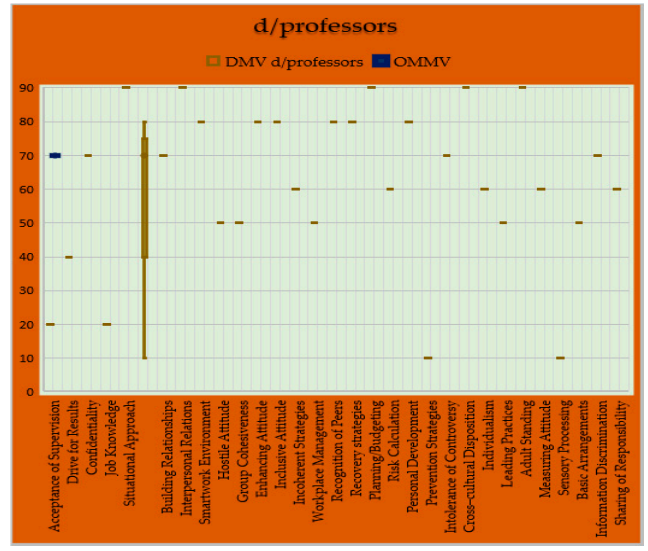

(b) DMVs for $\mathrm{d} /$ professors

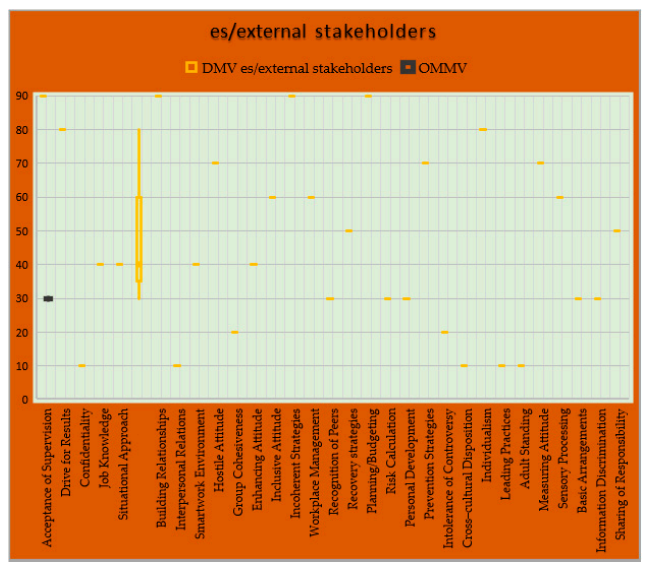

(d) DMVs for es/external stakeholders

Figure 3. Dimensional modal value (DMV) that the four university groups used to scale the pictures and definitions of the chosen test based on their qualitative scores ( $q s)$. The left bar of a different color represents the overall modal value of the total matrix of the scores in each group (OMMV). Note: all scores are dummies generated in $10 \%$ of each quota sample through a RAND().exe formula.

\section{Materials and Methods}

The integration of radically different orientations was at the heart of this research, where each step could have theoretically led elsewhere (at least in the conceptual analysis). The design was experimental, so considerable bias could have affected the measurements and distorted the information transmitted in this paper.

For many authors, intentions are not only influenced by attitudes but also by social (normative) pressures. Therefore, the ultimate determinants of all behaviors are behavioral reactions to normative experiences based on others [31]. However, our model for reacting to an unexpected event (such as an emergency) was not focused on acquiring knowledge filtered by information that does not offer solutions or adaptive planning processes, but instead on a retroactive approach that separated the two inputs:

- The function of the membership matrix of the three other groups of organizational actors of the sampled university;

- The organizational effectiveness function of the general system. 
For this, we considered the four main groups of a university (students, teachers/professors, administrative staff, and external stakeholders) using the following equation:

$$
\left[D M \forall\left(\begin{array}{ccc}
s & \cdots & d \\
\vdots & \ddots & \vdots \\
a & \cdots & e s
\end{array}\right) t \geqslant e_{i}\right]
$$

where DM is the decision-making $\forall$ (for all) relational matrices of $s$ (students), $d$ (teachers/professors), $a$ (administrative staff), and es (external stakeholders) within the time $t \geq \cap$ following $e_{i}$ (the emergency event).

The dynamics of the emergency-intervention-decision sequence gave rise to new problems, such as whether we are studying the pre-eminence of the individual or the system. Are there functional outcomes in one or more groups structurally combined at the university that can produce different structural university environments?

In addition, we must ask, will the ethical-moral mindsets of students-whether teachers, administrative staff, or stakeholders-be equal the effectiveness of the choices made? Moreover, in one or more structurally combined groups, will a perceived status of discordant cognitive imbalance be reduced by a stronger harmony triggered by each group in a different context? If this harmony can be observed in all existing systems in the sense that it can be found in all observed systems, will a heuristic value prevail over the other characteristics currently designed by our university models? Are the global emergency space and the organizational university space sufficiently filled with decision-making images? Is there an awareness that self-organization and self-management can weaken the strength of continuous communication between the four groups?

Because the dimensions were designed by the first researcher, who is a scholar in systems theory, it was difficult to guess whether the respondents would have willingly performed the graphical test following the step by step inputs furnished by the researcher. On the one hand, the dimensions were clearly connected in many examples, which indicated overlapping meanings. However, they were also blurry sometimes, which suggested that the interval scales simply measured the frequency of the respondents' choices and not the suitability or unsuitability of one dimension compared to another. On the other hand, the selection, sampling, and statistical model provided by the researcher also had transferable meaning: this model was used each time to ask questions about the decision-making models and their feasibility in the occurrence of emergency events.

Clearly, our respondents were neither "riskless" nor expected to maximize their expected utility. Since this situation was experimental, the goal of action could not be defined as simply hedonistic. There were complexities in the interlacing of choices in scoring pictures and definitions in the graphical test, which introduced an indifference curve and gradually restructured the classical utility theory that we wish to express through our analysis. We will return to this point in the conclusion.

\section{Discussion and Results}

The visual stimuli in the pictures used to test respondents may have generated reflections on the functionalist conception of the mind, which describes an awareness of the self-representation of things, occurrences, and thoughts, as well as the delivery of information drawn from the organizational capacity useful for controlling or planning behavior-see Figure 4 below.

A1. A random university matrix (RUM0) was constructed by randomly sampling the class scores by $\mathrm{OE}$. The university matrix filtered by the environment (EFUM0) was constructed by sorting the scores by their model dimension (DMV) in decreasing order compared to the model values of the total OMMV matrix. 


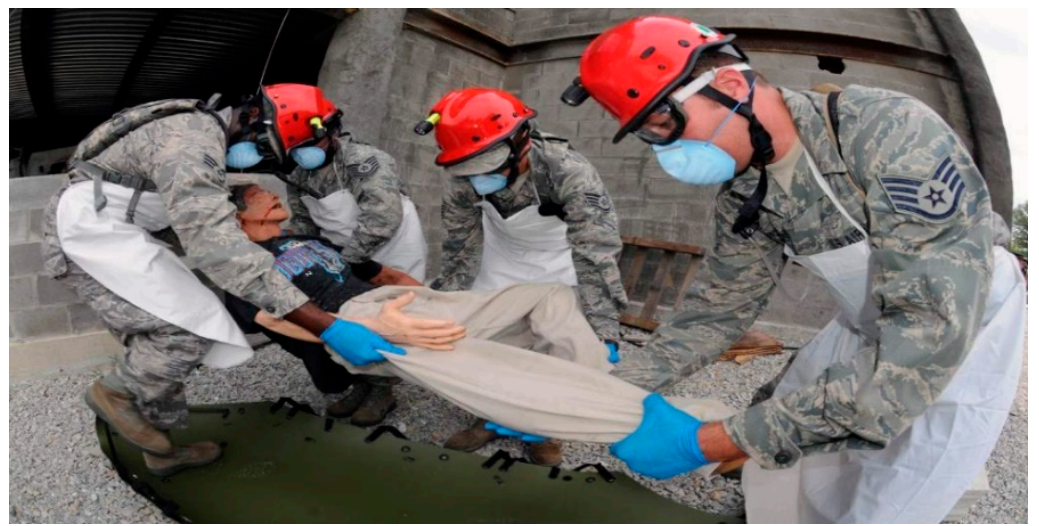

Figure 4. An image of disaster-recovery capability that stresses the importance of cooperation and a timely prevention response to an unexpected event, available at: https://media.defense.gov/2011/Dec/ 31/2 000188925/-1/-1/0/110818-F-9999C-100.JPG.

We, therefore, argue that certainty in choices implies "deterministic knowledge". In turn, risk entails "complete probabilistic knowledge". Uncertainty requires "partial probabilistic knowledge", and ignorance requires "non-probabilistic knowledge" [32-34]. The decision framework is, therefore, an aspect of choice that we must consider as mixed for our university respondents, who must test the OE dimensions under the following conditions: (1) certainty that it is a test; (2) the risk that something unexpected may happen; (3) uncertainty over the position of the other groups of respondents; and (4) ignorance of the event that could possibly occur. The decision framework $(D F)$ when submitting the BARS test is as follows: certainty $(C)$ plus risk $(r)$ plus uncertainty $(U)$, plus ignorance (i): $D F=C+r+U+i$. We already specified that time is a variable that cannot be calculated accurately. The respondent assessment mechanism should self-assess risks and exclude them from the potential preferential rewards. The relative attractiveness of the choices in the test does not depend on the other choices that were drawn from the respondents in other groups who know that their choices do not improve the score. Indeed, people are often eager to choose scores with the promise of gratifying results, but our respondents were alerted that the test scoring points provided no indication of the respondent's ability. This can be formalized as follows:

A2. For any $\mathrm{OE}$ dimension, let $D M_{s}=x_{1} ; D M_{d}=x_{2} ; D M_{a}=x_{3} ; D M_{e s}=x_{4}$. Suppose we also have $d m v_{1}=d m v_{2}=d m v_{3}=d m v_{4}$. Each $s, d$, a, es under the path independence formula for the four university groups is more complicated, but also more typical because it represents what cannot be excused or compensated.

Suppose also that we cannot infer the DMVs or the OMMVs to reasonably express or satisfy the parameters in the sample test. The visual representations of the dimensions highlight the intentionality of the choice in the face of an emergency event: They represent a state of affairs that is neither intentional nor representative until the respondent is not aware of being able to assess his/her own organizational effectiveness. This can be formalized as follows:

A3. For any $\mathrm{OE}$ dimension, let $10=90$. Suppose we also have $10 \neq 90$. Suppose also that $10 \cong 90$ and that $s, d, a$, es prefer to define and record the variables in the test in the same way. Here, we have a conditioned stimulus for the four groups, which are apparently wary of the design between the groups and the models that were created to describe the decision-making choices (i.e., the individual and organizational models).

Even if the properties of the dimensions rely on rationality, the "appeal-to-choice" models suppose the independence of irrelevant alternatives. Moreover, the existence of conditionality based on the permanence of respondents in the university could violate the principle of limited rationality. But, what about the parameters of the unattractive alternatives that could develop under the pressure 
and incompatible behaviors from other groups? Would the decision-making process then be stochastic, proving that $\ll 0 \leq x(M) \leq \sum_{x \in M} x(M)=1$ for all $x$ and all $M>>$ is true? [35].

Suppose that the ten events, $E_{1}, E_{2}, \ldots E_{10}$, are equally probable and that the wagers $w^{1}, w^{2}$, and the $w^{3}$ associated rewards (integers representing the amounts of money) have outcomes: see Table 2 below:

Table 2. Example of a decision-making process is stochastic by G.M. Becker (1963).

\begin{tabular}{ccccccccccc}
\hline & $\boldsymbol{E}_{\mathbf{1}}$ & $\boldsymbol{E}_{\mathbf{2}}$ & $\boldsymbol{E}_{\mathbf{3}}$ & $\boldsymbol{E}_{\mathbf{4}}$ & $\boldsymbol{E}_{\mathbf{5}}$ & $\boldsymbol{E}_{\mathbf{6}}$ & $\boldsymbol{E}_{\mathbf{7}}$ & $\boldsymbol{E}_{\mathbf{8}}$ & $\boldsymbol{E}_{\mathbf{9}}$ & $\boldsymbol{E}_{\mathbf{1 0}}$ \\
\hline $\boldsymbol{w}^{1}$ & 3 & 1 & 4 & 2 & 2 & 5 & 3 & 4 & 4 & 2 \\
$\boldsymbol{w}^{2}$ & 4 & 2 & 4 & 3 & 2 & 5 & 3 & 4 & 5 & 2 \\
$\boldsymbol{w}^{3}$ & 2 & 4 & 2 & 4 & 5 & 1 & 4 & 3 & 2 & 3 \\
\hline
\end{tabular}

For every event, the reward from $w^{2}$ is at least as large as the reward from $w^{1}$. Hence, $w^{2}$ dominates $w^{1}$ and is preferable to $w^{1}$. Since the rewards from $w^{3}$ are simply a permutation of the rewards from $w^{1}$ (and all $E_{1}$ are equally probable), $w^{3}$ and $w^{1}$ are interchangeable. Hence, $w^{2}$ is also preferable to $w^{3}$. In an actual experiment, a subject might note that $w^{2}$ dominates $w^{1}$ and that he or she would never choose $w^{1}$. However, since $w^{3}$ yields larger rewards than $w^{2}$ for some events, a subject might at times choose $w^{3}$ over $w^{2}$ [35].

Difficulties appeared when examining the inferences from the choices. Suppose that all respondents marked their 33 DMVs on the OE dimensions-see Table 3 below:

Table 3. Example of a distribution of scores are all equal.

\begin{tabular}{cccccccccc}
\hline & $\boldsymbol{O} E_{\boldsymbol{1}}$ & $\boldsymbol{O} E_{\mathbf{2}}$ & $\boldsymbol{O} E_{\mathbf{3}}$ & $\boldsymbol{O} \boldsymbol{E}_{\mathbf{4}}$ & $\boldsymbol{O} \boldsymbol{E}_{\mathbf{5}}$ & $\boldsymbol{O} \boldsymbol{E}_{\mathbf{6}}$ & $\boldsymbol{O} \boldsymbol{E}_{\mathbf{7}}$ & $\boldsymbol{O} \boldsymbol{E}_{\mathbf{8}}$ & $\boldsymbol{O} \boldsymbol{E}_{\boldsymbol{X}}$ \\
\hline$s$ & 90 & 90 & 90 & 90 & 90 & 90 & 90 & 90 & 90 \\
$d$ & 90 & 90 & 90 & 90 & 90 & 90 & 90 & 90 & 90 \\
$a$ & 90 & 90 & 90 & 90 & 90 & 90 & 90 & 90 & 90 \\
es & 90 & 90 & 90 & 90 & 90 & 90 & 90 & 90 & 90 \\
\hline
\end{tabular}

Does this suggest that all four groups have severe linear apathy in the scoring dimensions for OE?

We must also ask ourselves whether the heart of this theory has a problem with the constraints and assumptions that in our introduction drew the "principle of a sure thing" in different directions. This possibility is plausible in the modeling of decisions, as the assumptions are meant to indicate the setting and not the environment. We cannot account for such hypotheses at this stage, since our partition of the set of scores according to their environment requires an alternative representation of the decision problem, and the respondents do not have the propensity to react to unknown factors.

In doing so, we assume that our quota samples are representative of the target population of interest and that the properties of the samples are reasonably precise enough to allow us to process an impartial cognitive mechanism of the respondents. We have sought to observe the strengths of large-scale cooperation, which is fully expressed in human groups, although this research does not explain when it is better or worse than competition during emergency dilemmas.

Thus, here we have a conditioning stimulus for the four groups, which are apparently distrustful of the design between the groups. Moreover, the models were created to describe the decision-making choices (i.e., the individual and organizational models). Borrowing from Luce's theory of probability, we developed a similarity $\mathrm{OE}$ university matrix among the results in descending order, restricting our attention to modal values, which, for whatever reason, were chosen in the test and demonstrate how the respondents in endowed academic institutions could satisfy behavior that is boosted by an environment filtered university matrix (EFUM0), which is integrated in relation to the individual contributions during the occurrence of an unexpected event [36].

This suggests a broad landscape reference for behavioral choices and decision-making other than the need to study a model, which could help explain whether collective rationality is adjusted in the face 
of a strong environmental emergency. Although we focused on the environment here, this approach not only uses natural point of view but also a mixture of social characteristics that can be traced in a limited decision-making process as it is integrated into institutional resources, organizational capacity, and the culture of adaptation arising from the structural environment in which the four university groups live in the same way.

Adaptation to the formation of habits in contexts of information asymmetry was an additional tool with key implications. If students had displayed a trend in scores among institutions, what would have happened for behavioral independence? Would this mean that staying at the university could generate persistent choice mechanisms?

\section{Limitations Bolster the Study: Looking Forwards}

A limitation of this model is that it was based on specific human parametric processes, which made it difficult to determine whether they were coincident with dynamic parameters, particularly the difference between the landscape of uncertainty and legal compromises, or with static behaviors, making them more like a generalized utility distribution representation.

Thanks to our method, we determined the variables that have the greatest impact on the choices of the respondents. Using these variables, we were directed towards the creation of alternatives, but the analysis of the alternatives did not predict the value of the maximum likelihood estimates for the choices of the respondents. The reasoning for the means, standard deviations, and scores in the quota sampling was motivated by an inductive approach and proven by a deductive approach. Each time, the discussion focused on the extendibility of decision-making issues, including a number of organizational characteristics, such as the formalization of actions, sources of authority, layers of undecidability, decentralization of practices, and the impact of socio-cultural variables on the style of each sub-group. Research has highlighted the importance of what organizational efficiency really means for the environment and its functional engineering.

We chose a circular regression for this study: (1) we stated the problem, then, (2) we tested the scores, until (3) second order decision problems arose statistically. Next, (4) we tried to refine the analysis, (5) rework the hypotheses, and (6) adjust our decision frameworks. The reasoning was reversed, as indicated in Section 2.1, simply because the researchers repeatedly returned to the same hypotheses, as the underlying constraints were not always representable as dependent on the environment or the framework of the respondents' decisions. The contexts and properties were all options, intrinsic to the appropriate environment that we declared "emblematic" for our research, but also to the relational representations of what is legitimately conceivable from an integrated measurement of a non-normative choice of university groups.

An immediate extension of our measurement is not possible at this stage. The observations, which were all collected in a mechanistic sample, can never provide the true sequences of the choices made by humans. In addition, if our research environment were a non-empty set, the images evaluated by the respondents, scored from 10 to 90 at intervals of 10, would have represented the importance of the variables in the object for an unexpected event of a fictitious nature.

This was not all the realization of a stochastic process that satisfies the utility as limited by the activities of researchers in science. It was subject to the environment and, at the same time, subject to an overcoat that could hide a network with several interconnections. It may be of interest to pure statisticians to refine our competitive recognition. What was desirable for our research could also constitute a misinterpretation of the data and a loss of validity. The explanatory power of science and its competitive recognition is represented by the ineffectiveness of its problems, in that science is responsible for such problems, as well as not responsible for an understanding, which in itself is exposed to failure. We stand by these conclusions and believe that we have achieved ideal progress thanks to the equalization of conditions, both in the situational and the organizational distribution of choices. We have satisfied a large number of hypotheses through our competitive recognition of the existence of science, and it is up to our colleagues to recognize this. 
Author Contributions: Data curation, R.F.; Formal analysis, R.F.; Investigation, R.F.; Methodology, R.F.; Project administration, R.F.; Resources, R.F.; Validation, R.F. and S.C.; Visualization, R.F. and S.C.; Writing-original draft, R.F. and S.C.; Writing-review \& editing, R.F. and S.C. All authors have read and agreed to the published version of the manuscript.

Funding: This research received no external funding.

Conflicts of Interest: The authors declare that there is no conflict of interest.

\section{References}

1. Miller, D. Two Ways to Think About Justice. Politics Philos. Econ. 2002, 1, 5-28. Available online: https://doi.org/10.1177/1470594X02001001001 (accessed on 6 January 2020).

2. ScienceDirect. Decision Theory. Topic-by-Topic Machine-Learning Approaches to Information. Available online: https://www.sciencedirect.com/topics/neuroscience/decision-theory (accessed on 30 April 2020).

3. Drabek, T.E. Revisiting the Disaster Encyclopedia. Int. J. Mass Emergencies Disasters 1999, 17, $237-257$.

4. Kreps, G.A. Disaster and the Social Order. Sociol. Theory 1985, 3, 49-64.

5. Merchant, K.A.; Van der Stede, W. Management Control Systems. Performance Measurement, Evaluation and Incentives; Pearson Education Limited: New York, NY, USA, 2007.

6. Sajo, A. Why do Public Bureaucracies follow legal rules? Int. J. Sociol. Law 1981, 9, 69-84.

7. Ostrom, E. Understanding Institutional Diversity; Princeton University Press: Princeton, NJ, USA, 2005.

8. Ostrom, E. Background on the Institutional Analysis and Development Framework. Policy Stud. J. 2011, 39, 7-27. Available online: https://pdfs.semanticscholar.org/cbcf/cf29ff30d31bc477bbf3f219a6c2037f7eb8.pdf (accessed on 6 January 2020).

9. Bammer, G.; Smithson, M. Uncertainty and Risk: Multidisciplinary Perspectives; Earthscan: London, UK, 2008.

10. Smithson, M. The Many Faces and Masks of Uncertainty 2008. Available online: http://citeseerx.ist.psu.edu/ viewdoc/download?doi=10.1.1.574.3193\&rep=rep1\&type=pdf (accessed on 6 January 2020).

11. Mortensen, C.D.; Ayres, C.M. Miscommunication; Sage Publications: Thousand Oaks, CA, USA, 1997.

12. Wieland, J.W. Responsibility for strategic ignorance. Synthese 2017, 194, 4477-4497. [CrossRef]

13. Georgetown University Medical Center. Why adults at risk for Huntington's choose not to learn if they inherited deadly gene. ScienceDaily. 16 May 2019. Available online: www.sciencedaily.com/releases/2019/05/ 190516103715.htm (accessed on 9 January 2020).

14. Georgopoulos, B.S.; Tannenbaum, A.S. A Study of Organizational Effectiveness. Am. Sociol. Rev. 1957, 5, 534-540.

15. Olsen, J.P. The Institutional Dynamics of the (European) University; Working Paper 15. Center for European Studies, University of Oslo: Oslo, Norway, 2005. Available online: https://www.sv.uio.no/arena/english/ research/publications/arena-working-papers/2001-2010/2005/wp05_15.pdf (accessed on 8 May 2020).

16. Fishburn, P.C. Nontransitive preferences and normative decision theory. In Decision Making under Risk and Uncertainty; Geweke, J., Ed.; Springer: New York, NY, USA, 1992; pp. 3-10.

17. Wikipedia, the Free Encyclopedia. Probability Distribution. Available online: https://en.wikipedia.org/wiki/ Probability_distribution (accessed on 9 January 2020).

18. Wallis, W.D. A Beginner's Guide to Discrete Mathematics; Springer Science \& Business Media: Berlin, Germany, 2003.

19. Grinstead, C.M.; Snell, J.L. Introduction to Probability. American Mathematical Society: Dartmouth, 2006; pp. 1-520. Available online: https://www.dartmouth.edu/ \{\}chance/teaching_aids/books_articles/ probability_book/amsbook.mac.pdf (accessed on 9 January 2020).

20. El Hami, A.; Radi, B. Incertitudes, Optimisation et Fiabilité des Structures; Lavoisier: Paris, France, 2013.

21. Gouran, D.S.; Hirokawa, R.Y. Functional theory and communication in decision-making and problem-solving groups: An expanded view. In Communication and Group Decision Making; Hirokawa, R.Y., Poole, M.S., Eds.; Sage Publications: Newbury Park, CA, USA, 1996; pp. 55-80. [CrossRef]

22. Putnam, L.L.; Stohl, C. Bona Fide Groups: An Alternative Perspective for Communication and Small Group Decision Making. Chapter 6; In Communication and Group Decision Making; Hirokawa, R.Y., Poole, M.S., Eds.; SAGE Publications Inc.: Newbury Park, CA, USA, 2012.

23. Corso, J.J.; Xu, C.; Whitt, S. Flattening supervoxel hierarchies by the uniform entropy slice. In Proceedings of the IEEE International Conference on Computer Vision, Sydney, Australia, 1-8 December 2013. 
24. Bolstad, W.M.; Curran, J.M. Introduction to Bayesian Statistics; John Wiley \& Sons: Hoboken, NJ, USA, 2016.

25. Paté-Cornell, E. Probabilistic Risk Analysis Versus Decision Analysis: Similarities, Differences and Illustrations. In Uncertainty and Risk. Mental, Formal, Experimental Representations; Abdellaoui, M., Luce, R.D., Machina, M.J., Munier, B., Eds.; Springer: New York, NY, USA, 2007; pp. 223-242.

26. Moscarola, J. Organizational Decision Process and ORASA Intervention. In Rethinking the Process of Operational Research and Systems Analysis; Tomlinson, R., Kiss, I., Eds.; Pergamon Press: New York, NY, USA, 1984; pp. 169-186.

27. Price, J. The Study of Organizational Effectiveness. Sociol. Q. 1972, 13, 3-15. Available online: www.jstor.org/ stable/4105817 (accessed on 9 January 2020).

28. Luhmann, N. Organisation und Entscheidung; Westdeutscher Verlag: Wiesbaden, Germany, 2000.

29. Ejimabo, N.O. The Influence of Decision Making in Organizational Leadership and Management Activities. J. Entrep. Organiz. Manag. 2015, 4, 138. [CrossRef]

30. European Commission/EACEA/Eurydice. The European Higher Education Area in 2018: Bologna Process Implementation Report; Publications Office of the European Union: Luxembourg, 2018; pp. 1-330. Available online: https:/eacea.ec.europa.eu/national-policies/eurydice/sites/eurydice/files/bologna_internet_0.pdf (accessed on 16 February 2020).

31. Fishbein, M.; Ajzen, I. Attitudes and opinions. Annu. Rev. Psychol. 1972, 23, 487-544. [CrossRef]

32. Susman, G.I.; Evered, R.D. An Assessment of the Scientific Merits of Action Research. Adm. Sci. Q. 1978, 23, 582-603. [CrossRef]

33. Hansson, S.O. Decision Theory. A Brief Introduction; Royal Institute of Technology (KTH): Stockholm, Sweden, 2005; pp. 1-94. Available online: https://people.kth.se/ \{\}soh/decisiontheory.pdf (accessed on 6 March 2020).

34. Etner, J.; Jeleva, M.; Tallon, J.-M. Decision Theory under Uncertainty. 2009. halshs-00429573v2. Available online: https://halshs.archives-ouvertes.fr/halshs-00429573v2/document (accessed on 6 March 2020).

35. Becker, G.M. Stochastic Models of Choice Behavior. Behav. Sci. 1963, 8, 41-55.

36. Luce, R.D. Individual Choice Behavior: A Theoretical Analysis; Wiley: New York, NY, USA; pp. 1-6. 